American Journal of Applied Sciences 1 (2): 76-83, 2004

ISSN 1546-9239

(C) Science Publications, 2004

\title{
Suppression of Instabilities and Stochastic Pulsation at Laser-Plasma Interaction by Beam Smoothing
}

\author{
${ }^{1}$ Frederick Osman and ${ }^{2}$ Heinrich Hora \\ ${ }^{1}$ School of Quantitative Methods and Mathematical Sciences, University of Western Sydney, \\ Locked Bag 1797, Penrith South DC 1797, Australia \\ ${ }^{2}$ Department of Theoretical Physics, University of New South Wales, Sydney, 2052, Australia
}

\begin{abstract}
The key problem of direct drive laser fusion is the appearance of parametric instabilities, stochastic pulsation, self-focusing (filamentation) and other anomalies. During the long years studies, the empirical and intuitively developed methods for smoothing of the laser beam were rather successful but a transparent understanding of the physics has still to be found. The first theory how the instabilities are reduced by smoothing was given recently by using PIG simulation while the suppression of the 10-picosecond stochastic pulsation by broadband laser beams was analyzed by the genuine two fluid models. A synoptic evaluation of these results is presented here where the correlation between the instabilities with the pulsation is evident. This opens new ways for direct drive laser fusion with the fundamental red laser light avoiding expensive and because of crystal defects unsolved problems with higher harmonic production.
\end{abstract}

Key words: Parametric instabilities, stochastic pulsation, self-focusing

\section{INTRODUCTION}

The generation of plasmas by lasers showed transparent classical properties only as long as the laser power was below a threshold $\mathrm{P}^{*}$ of about one megawatt. Only then the plasma generated by the laser resulted in the heating of the plasma to temperatures of few $10,000 \mathrm{~K}$ and emission of ions of few EV energy and electron emission similar to thermionic emission with space charge limited current densities. What happens above $\mathrm{P}^{*}$ was a number of anomalies causing many difficulties against direct drive laser fusion as generation of energetic ( $\mathrm{keV}$ to $\mathrm{GeV}$ ) ions of high charge number $\mathrm{Z}$, whose energy is linearly increasing on $\mathrm{Z}$ showing a nonthermal origin, as energetic $\mathrm{x}$-ray emission ("hot" electrons), as higher harmonics generation, as parametric instabilities, as filamentation by Pondero-motive and relativistic self focusing, as double layer effects and as the pulsating interaction seen from changes of the reflectivity between few percent and above $90 \%$ in stochastic sequence of about 10 to 30 ps. For direct drive laser fusion, these anomalous phenomena are most disadvantaged and during the last years only, some understanding was possible and how to control the phenomena ${ }^{[2,3]}$ based on computations with the genuine two-fluid model ${ }^{[2,3]}$. This was confirmed only very recently ${ }^{[1]}$ based on a detailed numerical analysis including microscopic theory proving how ultrabroad bandwidth laser beam smoothing suppresses parametric instabilities. One empirically developed method without deeper theoretical understanding was the beam smoothing of the laser pulse ${ }^{[4,5,6,7]}$. In view of this recent success ${ }^{[1,2]}$, a deeper recollection of the otherwise rather hidden experimental and theoretical work is presented here where the new aspects are proposed to focus on the very early work of ${ }^{[8]}$. It is explained here how the smoothing will lead to a suppression of the stochastic pulsation (stuttering interaction) as an alternative mechanism where, however, a link and perhaps a mutual interrelation is well possible since both mechanisms are based on the nonlinear (ponderomotive) force. The unified foundation of the parametric instabilities as derived from $^{[9]}$ was based on the nonlinear force theory ${ }^{[10,11,12]}$ while the relation with smoothing and stochastic pulsation is given by the nonlinear force was shown in several steps ${ }^{[2]}$.

From the broad stream of laser-plasma interaction work some less considered phenomena are highlighted ${ }^{[13,14,15]}$ for discussing the smoothing and the pulsation $^{[16,17]}$ phenomenon. The experiment by ${ }^{[15]}$ is then used as a visible example to explain the filamentation as well as the pulsation mechanism and how this all can be overcome by smoothing using the random phase plate ${ }^{[6]}$ while the action of broadband laser irradiation in suppressing the pulsation ${ }^{[2,3]}$ was demonstrated numerically before. This all indeed has to be recognized in a synoptic way together with the recent results of the suppression of parametric instabilities by broadband laser beam smoothing ${ }^{[1]}$.

The initial motivation for introducing smoothing was the suppression of filamentation ${ }^{[18]}$. Another much bigger problem of laser-plasma interaction was seen in the parametric instabilities ${ }^{[19]}$. What was not predicted, however, was that the experiments with smoothing resulted in an enormous reduction of these instabilities ${ }^{[13,20]}$ and where no clear theory was offered until recently ${ }^{[1,2,3]}$. On top of this it was clarified that the 
main evil with the anomalies at laser plasma interaction may not so much be instabilities but the stochastic pulsation. The reason for this complication consists in the fact that-at sufficiently high laser intensities-the laser light produces self-generated von-Laue diffraction gratings which changes the optical response of the plasma from a mirror reflection into a phase reflection and vice versa. This was noted numerically ${ }^{[18]}$ and demonstrated in experiments very convincingly later ${ }^{[17]}$. A numerical analysis showed this pulsation in all details $^{[3]}$ and on top it demonstrated that appropriate smoothing can suppress the generation of the standing wave produced diffraction gratings with their phase reflection. A crucial experiment could show before, how the pulsation could be suppressed by smoothing ${ }^{[13]}$.

It turns out that the smoothing is essential for laser fusion and that the main difficulties with laser fusion $^{[19,20]}$ can be eliminated. As a consequence of laser fusion it seems to be possible to work with the fundamental (red) frequency of the laser beams having then more than 3 times more energy in the laser pulse and avoiding the very expensive more than meter wide single crystals for higher harmonic generation of the laser beams ${ }^{[2,19,20]}$. The problems with these crystals are in the large number of crystal defects ${ }^{[21]}$, which are very high if the crystals are grown very fast.

The initial motivation for the technique of beam smoothing: Optical self-focusing (or filamentation) of laser beams in plasmas was discussed since the selffocusing phenomenon was observed in condensed materials (liquids and solids) where the threshold of a laser power $\mathrm{P}^{*}$ was characteristic, which was first, derived by ${ }^{[22]}$ on the basis of the nonlinear extension of the dielectric constant. For plasmas, the first result of the threshold-after self focusing has been measured ${ }^{[23]}$ was derived from the fact that the laser beam expels plasma from its center by the radial gradient of the time averaged electric field $\mathrm{E}$ of the beam:

$\mathrm{f}_{\mathrm{NL}}=\left(\mathrm{n}^{2}-1\right) \nabla \mathrm{E}_{\mathrm{o}}^{2} /(16 \pi)$

where, Eo is the amplitude of the optical field oscillating with a radian frequency $\omega$ and where $n$ is the complex refractive index in the plasma with an electron density ne determined by Langmuir's plasma frequency $\omega_{\mathrm{p}}$ :

$\mathrm{n}^{2}=1-\left(\omega_{\mathrm{p}}{ }^{2} / \omega^{2}\right) /(1-\mathrm{i} v / \omega)$ with $\omega_{\mathrm{p}}{ }^{2}=4 \pi \mathrm{e}^{2} \mathrm{n}_{\mathrm{e}} / \mathrm{m}$

$\mathrm{e}$ is the charge and $\mathrm{m}$ the mass of the electron and $v$ is the electron-ion collision frequency in the plasma, which causes the damping of the electromagnetic wave similar to the metal optics.

The theory of self-focusing on the plasma $^{[9,18,24,25,26]}$ included the compensation of the nonlinear or pondero-motive force (1) by the force of the gas dynamic pressure acting against the expelling of the plasma from the laser beam. Further the condition of total reflection of the beam lets by the radial varying refractive index due to the variation of the plasma density and further the condition of self diffraction of the laser beam had to be added to arrive at the threshold $\mathrm{P}^{*}$ (in Watts) of self-focusing in the plasma including the Rayleigh factor of 1.22 for a beam instead of a factor 1 for a slit ${ }^{[18]}$ depending on the plasma temperature $\mathrm{T}$ (in $\mathrm{eV}$ ) and the electron density given by the plasma frequency $\omega_{\mathrm{p}}$, Eq. (2) as:

$\mathrm{P}^{*}=1 \times 10^{6} \mathrm{~T}_{-}{ }^{5 / 4}$ for $\omega_{\mathrm{p}}-\omega$

$=8 \times 10^{3} \mathrm{~T}$ for $\omega_{\mathrm{p}} \gg \omega$

Since the laser produced plasmas have temperatures at least of few $\mathrm{eV}$, the self-focusing begins from laser powers of about MW.

This megawatt threshold $\mathrm{P}^{*}$ has a crucial importance of laser interaction with plasmas. Below $\mathrm{P} *$, the interaction works fully classically with generation and heating of the plasma to temperatures of few $\mathrm{eV}$. This is normally the range of the industrial use of lasers for welding or cutting. Above $\mathrm{P}^{*}$ it was observed that the emitted ions had energies ${ }^{[27]}$ up to $10 \mathrm{keV}$ and electron emission current density was more than 1000 times higher ${ }^{[28]}$ than permitted by space charge limitation. These observations were the first unexpected anomalies, which were confusing the whole field of high intensity interaction of lasers with materials producing plasma.

It turned out that the self focusing is the reason causing a shrinking of the laser beam to such diameter (for $\mathrm{P}=10 \mathrm{MW}$ measured by ${ }^{[23]}$ of 3 to $5 \mu \mathrm{m}$ ) such that the laser intensity exceeds about $10^{13} / \lambda^{2} \mathrm{~W} / \mathrm{cm}^{2}$ (laser wave length $\lambda$ in $\mu \mathrm{m}$ ) where the nonlinear electro dynamic forces in the plasma dominate against the thermo kinetic forces. The laser forces accelerated the electron cloud and the ions follow separated by the charge number $\mathrm{Z}$ of the ions as observed from the linear Z-dependence of the ion energy. This all could be based on the general formulation of the force density in plasmas ${ }^{[29]} \mathrm{f}=\mathrm{f}_{\mathrm{th}}+\mathrm{f}_{\mathrm{NL}}$ consisting in the thermo kinetic force $f_{\text {th }}=-\nabla P$ is given by the gas dynamic pressure $P$ and the general nonlinear force ${ }^{[12]}$ :

$\mathrm{f}_{\mathrm{NL}}=\mathrm{j} \times \mathrm{H} / \mathrm{c}+\mathrm{E} \rho+\mathrm{P} \bullet \nabla \mathrm{E} / 4 \pi+(1 / \omega)(\partial / \partial \mathrm{t}) \mathrm{E} \nabla \bullet\left(\mathrm{n}^{2}-1\right)$

$\mathrm{E} / 4 \pi(1+(1 / \omega) \partial / \partial \mathrm{t})\left(\mathrm{n}^{2}-1\right) \mathrm{E} \bullet \nabla \mathrm{E} / 4$

Here one recognizes on the right hand side first the Lorentz term $f_{\text {Lorentz }}=\mathrm{j} \times \mathrm{H} / \mathrm{c}$ to the plasma current density $\mathrm{j}$ and the vacuum velocity of light $\mathrm{c}$, then the Coulomb term E $\rho$ with the electric charge density $\rho$ and as the third term the Kelvin Pondero-motive term (see Eq. (1) $\mathrm{Of}^{[12]}$ ):

$\mathrm{f}_{\text {Kelvin }}=\mathrm{P} \bullet \nabla E / 4 \pi=\left(\mathrm{n}^{2}-1\right) \nabla \mathrm{E}^{2} / 8 \pi-\left(\mathrm{n}^{2}-1\right) \mathrm{E} \times(\nabla \times \mathrm{E}) / 4 \pi$

The remaining terms in Eq. (4) are new nonlinear terms, which were derived from the general equation of 
motion in plasmas from the studies of laser interaction. The proof ${ }^{[29]}$ for the final generality of Eq. (4) was given by momentum conservation for the non-transient case $(\partial / \partial \mathrm{t}=0)$ and for the transient case of symmetry of the terms ${ }^{[29]}$ and from the gauge and Lorentz invariance $^{[30]}$. The success in applying of this nonlinear force for the acceleration of electrons by lasers in vacuum was shown in details ${ }^{[31,32,33,34]}$. For the correct interpretation it is necessary to mention that Kelvin's Pondero-motive force is identical with the nonlinear Schlüter term ${ }^{[35]}$ :

$\mathrm{j} \bullet \nabla\left(1 / \mathrm{n}_{\mathrm{e}}\right) \mathrm{jm} / \mathrm{e}^{2}=\left(\omega_{\mathrm{p}}{ }^{2} / \omega^{2}\right) \mathrm{E} \bullet \nabla \mathrm{E} / 4 \pi$

remembering the definition of the electric polarization $\mathrm{P}$ and Eq. (2) without collisions:

$P=\left(n^{2}-1\right) E / 4 \pi$

From Kelvin's Pondero-motive force (5) follows formally an expression of the "field gradient force" (1) what led to the common expression of "Pondero-motive force" for (1). As is known about (plane wave) perpendicular incidence of laser radiation on a plasma $^{[10,12]}$, the Schlüter term is then zero. Nevertheless there is a force of the form of Eq. (1). In this case, however, the nonlinear force $f_{N L}$ is the result of the Lorentz term in Eq. (4). This confusion of the definitions is avoided if one uses the general expression of the nonlinear force (4) for the electro-dynamic part of the force density in a plasma. If a laser beam has local intensity maxima, the so called hot spots, which are produced by dielectric nonlinearities especially in solid state laser amplifiers or optical glass components even if single mode laser oscillators are used, then it is necessary to avoid this no uniform irradiation for special cases as for laser fusion. This was the motivation to invent the smoothing techniques.

Beam smoothes techniques: Very disadvantageous phenomena were observed at high intensity laser irradiation of solid targets as seen from the mentioned $^{[27]}$ high energy Z-separated ion emission and very high current densities also of electron emission, from X-ray emission spectra indicating a number of temperatures and from backscattered spectra of the fundamental laser frequency or of its harmonics. Some reduction of these anomalies was first observed by the experiments of ${ }^{[5]}$ when laser irradiation had a broad spectrum, just using the broadband backscattered light from a laser irradiated target as the source of irradiation. This motivated then Deng to use a fly-eye smoothing where a plate with an array of a large number of little lenses was put into the laser beam before irradiation of the target. A reduction of the anomalies was observed. It should be noted that ${ }^{[36]}$ even in his early Kalmar laser system had used a kind of a phase correction plate as discussed in view of an earlier patent ${ }^{[37]}$ which may have led to some reduction of the anomalies.

The convincing breakthrough for smoothing came to the random Phase Plate (RPP) ${ }^{[6]}$ and the Induced Spatial Incoherence (ISI) ${ }^{[4]}$. The random phase plate consists in a glass plate where there is some roster or pattern with evaporated plane parallel dielectric material of different thickness of each part. This causes that the laser beam is split into a large number of beam lets each of which is phase shifted against the neighbor beam let. The plate is introduced into the laser beam at the end just before being focused to the target. The ISI requires that the laser oscillator receives a temporal incoherence e.g., of 2 ps at the beginning of the beam chain before going to the amplifier system.

The results are seen from an example comparing the laser intensity across a diameter with no smoothing, with RPP and ISI before the application to a target ${ }^{[13]}$. It should be noted that the RPP case may not have been the best achievement. Varying the roster finesse may have arrived at a different result. An example of a different roster structure will be shown in the following. Despite the possible non-optimized RPP, the effect caused by the smoothing is exceedingly significant as shown in Fig. 1 with the reduction of the backscattered $3 / 2$ th harmonics down to nearly one thousand of the unsmoothed case.

It is the standard assumption that the 3/2th harmonics emitted from a laser produced plasma is due to parametric instabilities, the stimulated Raman scattering SRS and the stimulated Brillion scatters SBS.

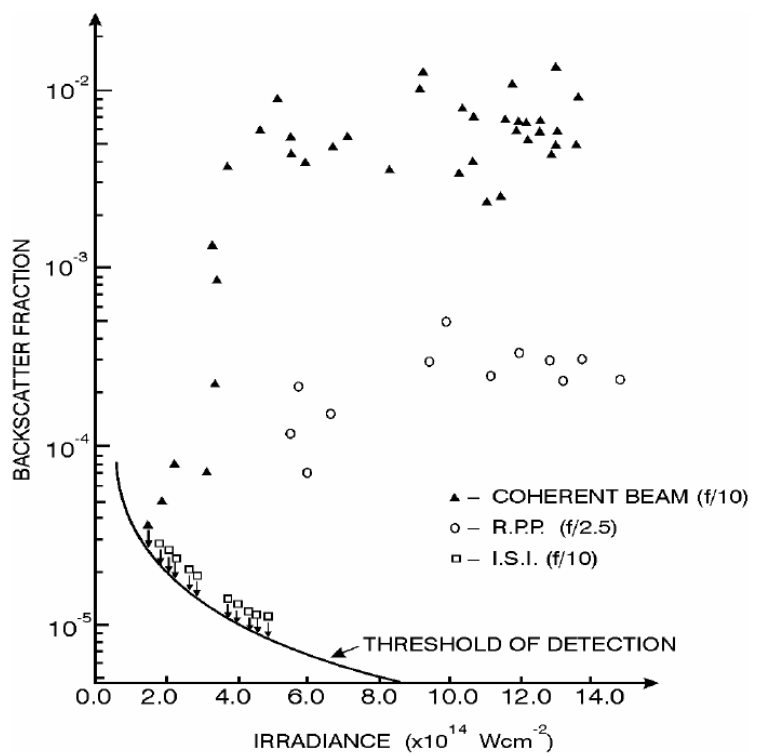

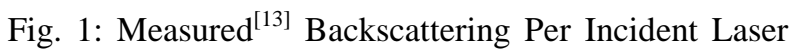
Power of the 3/2th Harmonized for Various Neodymium Glass Laser Intensities Without Smoothing (Coherent) and with Smoothing Using RPP or ISI 


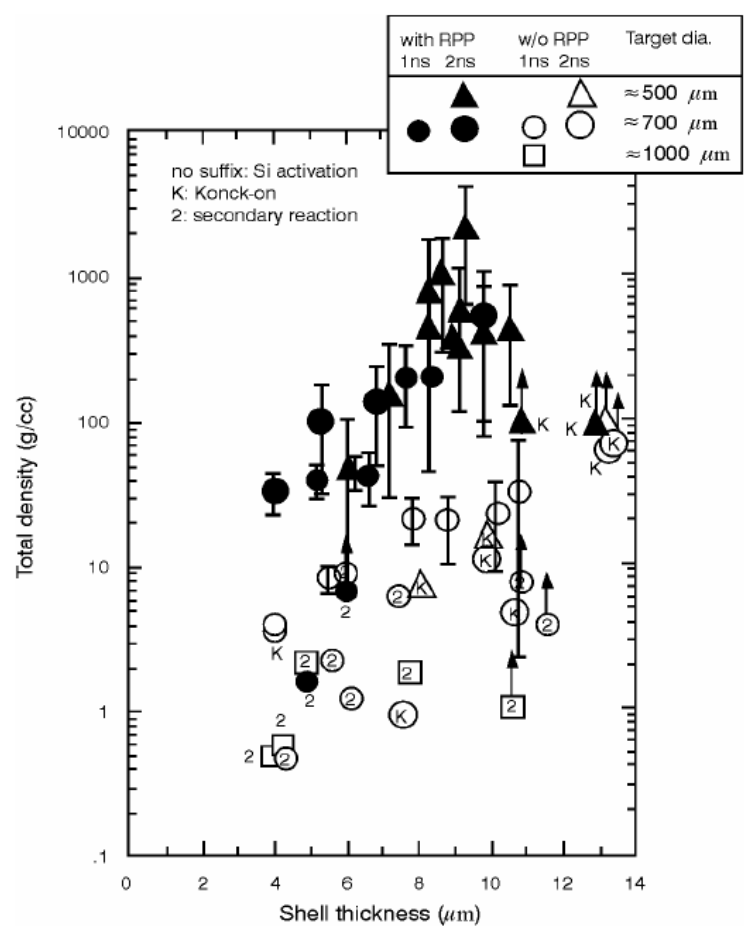

Fig. 2: Measured Laser Compression of Deuterated Polyethylene with Tritium Content of $1 \mathrm{G} / \mathrm{Cc}$ Solid State Density Depending on the Thickens of the Laser Irradiated Spherical Shell of Different Target Diameter ${ }^{[14]}$ without and with Laser Beam Smoothing Using the Random Phase Plate RPP

When all the above mentioned extreme anomalies of laser plasma interaction appeared, most attention was given to the suggestion that these were due to the instabilities and whole libraries were filled with speculations and comparison with unexplained experiments. One of the possible alternatives was an explanation of higher harmonic generation by electric double layers as a result of the genuine two-fluid model computations ${ }^{[38,39]}$. There is indeed a generation of higher harmonics ${ }^{[40,41]}$ causing a resonance in the supercritical range of the plasma density even at perpendicular incidence (contrary to the FörsterlingDenisov resonance absorption). Furthermore the second harmonic generation measured by ${ }^{[42]}$ even at very low plasma densities could be explained by this theory and not by the parametric instabilities which are restricted to certain specific densities only.

It should be mentioned that further smoothing schemes e.g., by the combination of RPP and ISI have been developed ${ }^{[7]}$. The generation of a broadband spectrum from the otherwise very narrow band of the coherent laser beam is an essential result of ISI and was developed also as "broad band smoothing" ${ }^{\text {"[3] }}$. Another observation was important by using the smoothing. It was observed $\mathrm{in}^{[13]}$, that irradiation of the target with the (unsmoothed) coherent laser light produced a stochastic pulsation of the $3 / 2$ harmonic emission ${ }^{[13]}$ with a duration of a few tens of a picosecond. When using the smoothing, the $3 / 2$ emission was not pulsating and was nearly continuously constant. This kind of a few tens of a ps pulsation was also seen from the Rogowski-coil diagnostics of the emitted plasma at nearly constant unsmoothed laser irradiation ${ }^{[44]}$. The same experiment was not performed with smoothing.

A very drastic example how the use of beam smoothing results in an increase of direct drive laser compression of plasma for fusion can be seen from the measurements of ${ }^{[14]}$ where the smoothing results in about ten times higher compression by using the random phase plate ${ }^{[6]}$ than without smoothing (Fig. 2).

Self-generated von-Laue-gratings and their Suppression by Smoothing: On top of the mentioned complications of laser-plasma interaction with the generation of ions with charge numbers of $\mathrm{Z}=58$ and energies up in the $\mathrm{GeV}$ range ${ }^{45]}$ and with anomalously high current densities, with ponderomotive ${ }^{[18]}$ and relativistic $^{[31,46,47]}$ self focusing, with suppression of higher harmonics by smoothing (Fig. 1) up to a factor 1000 , there seems to be another phenomenon of a key importance, the stochastic pulsation with a sequence in the $10 \mathrm{ps}$ range as is shown ${ }^{13]}$. It's incidentally observed suppression by smoothing ${ }^{[13]}$ may be not so much related to instabilities but may be a process of selfgenerating von-Laue gratings and their thermal washing out followed by another grating generation and again washing out etc.

The first indication of these difficult mechanisms in laser-plasma interaction was seen in numerical studies ${ }^{[18]}$ in 1974 what was the basis for finding out of this dilemma by understanding how the laser beam smoothing can suppress the stochastic pulsation. It all began with the computation of the plane geometry interaction of laser radiation with plasmas including the nonlinear force where the optical properties were carefully taken into account based on the usual optical absorption and on the correct (contrary to the Silin-Max approximation) nonlinear optical constants and the Maxwell an exact wave field with appropriate use of the "local reflection". Irradiation of a $2 \times 10^{14} \mathrm{~W} / \mathrm{cm}^{2}$ neodymium glass laser intensity on a linearly increasing ramp of deuterium plasma of $50 \mu \mathrm{m}$ thickness of the first stage A, Fig. 3, resulted in a penetration of the laser light to the critical density where this was reflected as a mirror reflection similar to the case of a metal with skin depth and (due to absorption) in the generation of a partial standing wave pattern. 2 ps later, however, when the fully coherently assumed laser intensity has grown to $2 \times 10^{16} \mathrm{~W} / \mathrm{cm}^{2}$, (B in Fig. 3) The light entered the very low plasma density but reached the critical density only after fading down to less than one hundred of the initial intensity. What has happened? The partial standing wave field had pushed the plasma toward the nodes of the standing wave and 
produced a density ripple which acted as an idealist, self produced von-Laue-Bragg grating for a nearly $98 \%$ phase reflection of the light even at the very low peripheral plasma density. One may say that such a one-Laue process is a Brillion process but we carefully have to avoid the word Brillion because since it is used in laser plasma interaction for the basically different (not hydrodynamic!) A microscopic instability process where laser radiation is converted into acoustic waves determined by wave vector relations.

This change from mirror to phase reflection was seen by Lubin in $1974^{[16]}$ from measuring the time resolved reflection of a laser-produced plasma. At the mentioned intensities, first the reflectivity was a few percent for few picoseconds, growing then to more than 90\% for several picoseconds, jumped then for few ps again to less than $10 \%$ and after further few ps again above $90 \%$ etc. The duration of the longest high reflecting periods was stochastically changing between some picoseconds and few tens of ps similar to stochastic pulsation later seen $\operatorname{in}^{[44]}$ or with the nonlinear force produced anomalous double layers ${ }^{[48]}$. Also the "question mark experiment" phase reflection with the reflected question mark pattern standing upside down. If this experiment would have been done with few ps time resolution and with detection of very low reflectivity, there would have been upright faint question marks between the strong upside-down cases stochastically changing within the mentioned several ps intervals.

This very complicated interaction process can be understood as seen numerically first in the cases of Fig. 3 (see more in the initial reference ${ }^{[18]}$, Section 7.5, or Fig. 2-3 of ${ }^{[12]}$. When, however, the phase reflection is cutting the optical penetration through the plasma corona, it happens that the density rippled plasma there is then gas dynamically relaxing and loosing the ripple within several ps such that then the laser light can penetrate again to the critical density and produce mirror reflection there. But then again the density ripple of the plasma corona occurs again with phase reflection etc. Exactly this has been reproduced numerically ${ }^{[2,3]}$. It shows also that the plasma corona as a whole block is getting a nonlinear force accelerated to the notorious velocities of $10^{7} \mathrm{~cm} / \mathrm{s}$ which acceleration ends each after few ps because of the mentioned density ripple and phase reflection until the thermal relaxation of the ripple permits another hit of the plasma corona to the mentioned velocities as seen from Fig. 4, lower part. The plasma corona is pushed to the velocity of about $10^{7} \mathrm{~cm} / \mathrm{s}$ within the first few ps. while the velocity ripple shows the motion of the plasma to the nodes of the standing wave field as seen in the energy density profile (upper part of Fig. 3). While the plasma then moved inertial, shifting the critical density to the plasma interior.

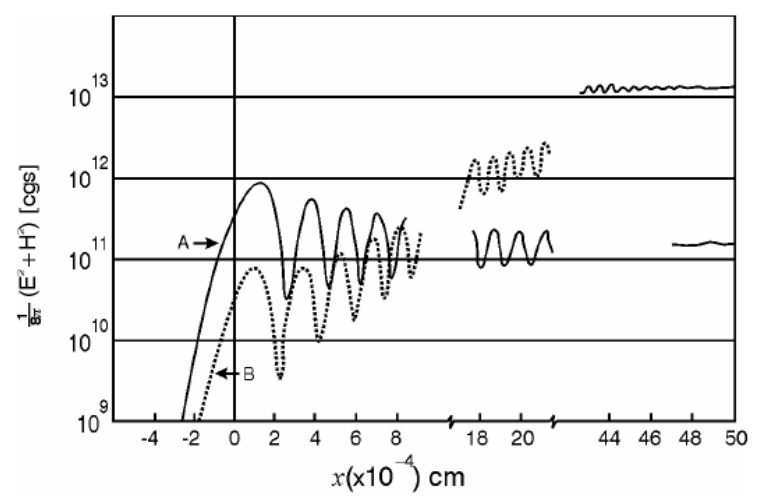

(a)

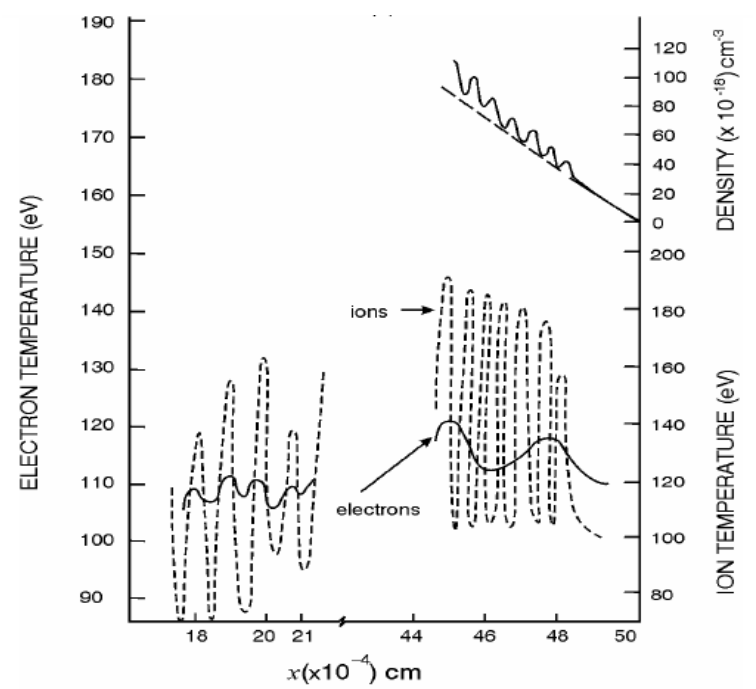

(b)

Fig. 3: (a) A Laser Beam Incident from the Right Hand Side on a Plasma of Initial Temperature of 100 $\mathrm{EV}$ and Linear Density Increasing from zero at $\mathrm{x}$ $=50 \mu \mathrm{m}$ to the Cutoff Density at $\mathrm{x}=0$ (where $\left.\omega_{\mathrm{p}}=\omega\right)$ and the Increasing More Rapidly. The Exact Stationary Time Dependent Solution Without Retardation of the Maxwell an Equations with the Nonlinear Refractive Index, Based on the Intensity Dependent Collision Frequency, Results in an Oscillation of the Electromagnetic Energy Density $\left(\mathrm{E}^{2}+\mathrm{H}^{2}\right) / 8 \pi$ due to the Partial Standing Wave and Dielectric Swelling of the Amplitude (curve A). At a Later Time (2 ps) the Laser Intensity is $2 \times 10^{16} \mathrm{~W} / \mathrm{cm}^{2}$ (curve B), where the Relative Swelling Remains, but the Intensity at $x=0$ is Attenuated by more than a Factor 100 due to the Phase Reflection of the Electromagnetic Wave by the then Density Ripple given by the Straight Line in the Upper Part of (b) which was Produced by the Nonlinear Force Pushing the Plasma into the Nodes of the Partial Standing Wave While Slowly Moving Hydrodynamic ally to Lower Density for the Dashed Line Initial Density. The Electron and Ion Temperatures are Increase Following the Ripple by Dynamic Compression at Conditions Identical to Curve B 


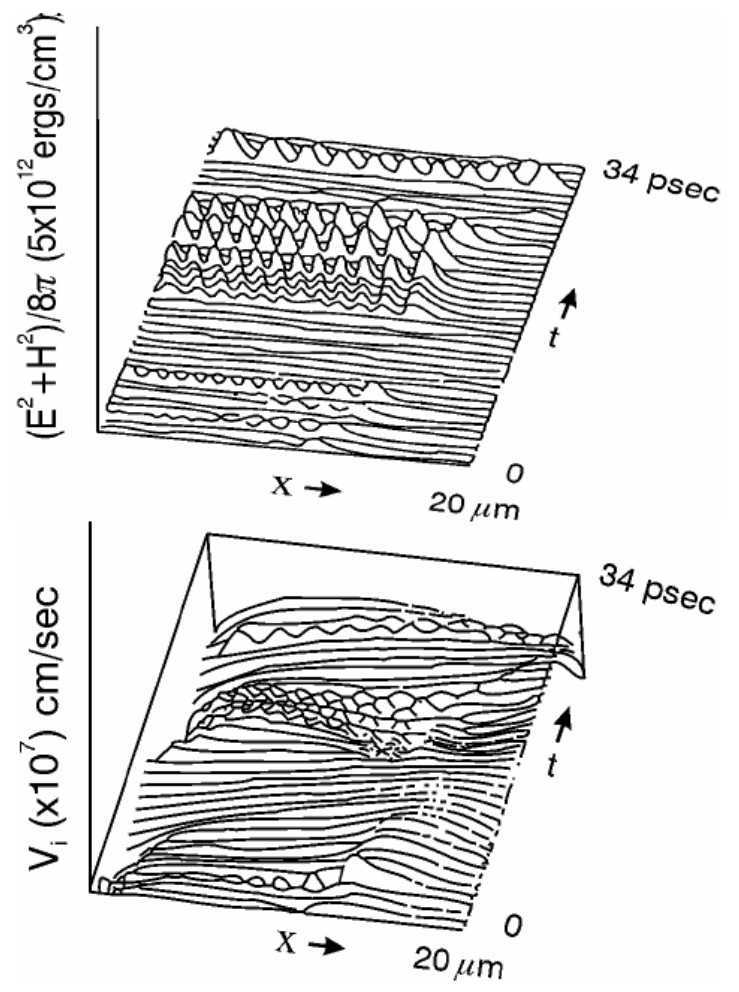

Fig. 4: Computation for Neodymium Glass Laser Irradiation with $10^{15} \mathrm{~W} / \mathrm{cm}^{2}$ Intensity on a Plasma Slab of $20 \mu \mathrm{m}$ Thickness and Initially Density Growing Linearly from 0.5 to 1.3 Times Critical Density and $100 \mathrm{eV}$ Temperature. The time Development in Steps of one ps of the Electromagnetic Energy Density of the Laser Field $\varepsilon=\left(\mathrm{E}^{2}+\mathrm{H}^{2}\right) / 8 \pi$ (a) and of the Ion Velocity vi Shows the Pulsation of Penetration or Stopping of the Laser Energy Synchronous with Ion Motion Whose Net Value Increases in Blocks after each Electromagnetic Interaction with the Corona ${ }^{[54]}$

The generated density ripple relaxes until about 20 ps when again the laser penetrates along the then monotonous and unrippled density profile to the critical density for mirror reflection, generating again a partial standing wave with pushing the plasma to the nodes as seen from the rippled velocity profiles and stopping the interaction after having given another push to the plasma corona by an additional $10^{7} \mathrm{~cm} / \mathrm{s}$ velocity.

This stochastically stuttering interaction was measured by ${ }^{[17]}$ by observing the ps pulsation of the acceleration of the plasma at laser irradiation see Fig. $4.14 \mathrm{c} \mathrm{of}^{[49]}$ while the pulsating acceleration of each plasma group for each about $10^{7} \mathrm{~cm} / \mathrm{s}$ was seen from the fully modulated spectrum at a narrow angle measured with glass fibers, Fig. 3.7 of $^{[49]}$. This experiment provides the key answer together with the theory (Fig. 4 of $^{[3]}$ why direct drive for laser fusion was nearly impossible during the preceding 20 years. This was shown also that the instabilities may not have been the reason for this dilemma but the $10 \mathrm{ps}$ stochastic pulsations (stuttering) process. The computations of the stuttering $^{[3]}$ interaction (Fig. 3) were extended to use a wide band laser irradiation, e.g. of $0.5 \%$ frequency width. It was then seen ${ }^{[17]}$ that this washes out the coherent density ripple and immediately results in a suppression of stuttering and an ideal high transfer of the laser radiation at a low reflectivity to the plasma corona as needed for direct drive laser fusion. This was seen before experimentally by using the intuitively suggested laser beam smoothing techniques with wide band laser radiation ${ }^{[4]}$.

\section{Experimental evidence of pulsation and suppression} by smoothing: Another experiment for a detailed proof that the smoothing was not only suppressing the filamentation as envisaged from the beginning-but is suppressing the much more important stochastic pulsation, was published by ${ }^{[15]}$. When the random phase plate for a $9 \mathrm{~cm}$ diameter laser beam had dielectric 2 $\mathrm{mm}$ squares for $180^{\circ}$ phase change, the pictures of spatially and temporally resolved plasma still showed an unsmooth result with beam-parallel filament structures. On top of this, the picture also showed structures perpendicular to the laser beam in about 40 ps distance clearly indicating the stochastic pulsation. But when using a random phase plate with $1 \mathrm{~mm}$ squares, rather smooth plasma was shown, where both the filamentation and the pulsation had disappeared.

For wave optics, the squares would have led to a focal spot diameter of $132 \mathrm{~mm}$ and $265 \mathrm{~mm}$ for the 2 $\mathrm{mm}$ respectively $1 \mathrm{~mm}$ squares. It is remarkable that in this case ray optics may be applicable where the filaments are squeezed into the $65 \mathrm{~mm}$ focal diameter of interaction. The beamlets of the squares are then about two wavelengths for the $2 \mathrm{~mm}$ and about one wavelength for the $1 \mathrm{~mm}$ squares. This is just what we expect from our density ripple calculations. As soon as neighbor filaments are out of phase within a wavelength distance or less, the washing out of any density ripple happens due to lateral interaction. For 2-wavelength distance the effect of washing out is too small. Indeed the addition of broadband as used by $\operatorname{SSD}^{[7]}$ may even better arrive at the necessary low reflectivity, nonpulsating, instability-suppressed and filamentless interaction as needed for the ideal direct drive laser fusion. This was considered in the past to be possible only with the 3 rd harmonics of the laser beam. The use of the fundamental wavelength should work similarly applying some modifications for the different conditions compared with the third harmonics. For the applied random phase plate a condition may be that the width $\mathrm{w}$ of the squares should be determined by the laser wavelength $\lambda$ :

$\mathrm{w}=\mathrm{LF} / \lambda$ 
where, $\mathrm{L}$ is the diameter of the lens equal to the laser beam or the random phase plate and $\mathrm{F}$ is the focus diameter of the laser beam at the plasma interaction.

Conclusions for laser-fusion in the NIF scale: The results about smoothing will provide an essential improvement for the conceptual design of laser operated direct drive fusion power stations. It will permit the operation with the red light from neodymium glass lasers without the need of the expensive frequency tripling by numerous single crystals of diameters in the scale of meters. Even for frequency doubling, the defects in too fast grown crystals may be unacceptable such that the only way out would be using the fundamental frequency with appropriate smoothing as elaborated here. The difficulties ${ }^{[19,20]}$ with the present billion dollar fusion lasers as NIF may well be solved along the present lines of design ${ }^{[50]}$, but for the next steps an inclusion of an appropriate smoothing for the red laser light may be considered.

For an estimation of fusion gains we may use here volume ignition ${ }^{[48,51]}$, which is, an easy "robust" compression scheme ${ }^{[52]}$ reaching nearly the same very high fusion gains as the very complex spark ignition. Using now the 4.5 MJ red laser irradiation ${ }^{[53]}$ of NIF with a hydrodynamic efficiency pessimistically of 5\% only, the total gain related to the laser energy is 14.5 at compression to 1000 times the solid-state density with an ignition temperature of $3.5 \mathrm{keV}$. At a compression to 3000 times the solid state, the total fusion gain is 35 and the initial temperature $2.9 \mathrm{keV}$. The total fusion gain of 35 would well be sufficient for a power station if lasers with more than $15 \%$ efficiency are available. The gains are also in some agreement with the above-mentioned indirect drive with third harmonics.

Nevertheless, the low hydrodynamic efficiency is rather poor though it may be then a feasible solution for the energy production without the climate catastrophe. It may not be the final solution since it may well be possible that the here described improvement may be overcome by a ten times higher efficient scheme for laser fusion, known as the fast ignitor ${ }^{[54]}$ but there are many details still to be clarified ${ }^{[51]}$.

A next step may consist in a more detailed study of the stochastic pulsation experiments and of parallel numerical studies using the genuine two fluid models ${ }^{[12,39]}$. Experimental tests may include whether the smoothing is resulting in continued low mirror reflection suppressing phase reflection. This can be done by direct studies of the reflectivity or by using the question mark experiment ${ }^{[8]}$ as a tool to check whether phase reflection has been eliminated or not. This is possible with a comparably low budget but may result in a considerably high gain for the expensive super lasers applied for laser fusion.

\section{REFERENCES}

1. Yanqing $\mathrm{Wu}$, Shenshang Han, Xianyang Song, Zhizhan Xu, 2001. Yuhui Tang and Bing Shuai: Plasma Physics and Controlled Fusion 43469.

2. Hora, H. And Aydin, M. 1999. Laser and Particle Beams, 17, 209.

3. Hora, H. And Aydin, M. 1992. Phys. Rev. A45, 6123.

4. Lehmberg, R.H. and S.P. Obenschain, 1983. Opt. Comm. 46, 27.

5. Xi-Ming Deng and Wenyan, Yu. 1984. Advances in Inertial Confinement Fusion, IAEA Technical Committee Proceedings, Yamanaka, C. Ed., Inst. Laser Engineering, Osaka Univ., p. 66.

6. Kato, Y., Mima, K., Miyanaga, N., Arinnga, S., Kitagawa, Y., Nagatsuka, M. And Yamanaka, C. 1984. Phys. Rev. Letters 53, 1057.

7. Skupski, S. et al. 1984. J. Appl. Phys. 53, 1057.

8. Pant, R.C., Eidmann, K., Sachsenmaier, P. and Siegel, R. 1976. Opt. Comm. 16, 396.

9. Chen, F.F., 1974. Laser Interaction and Related Plasma Phenomena, H. Schwarz et al. Eds. Plenum, New York, 3A, 291.

10. Hora, H. 1969. Physics of Fluids, 12, 181.

11. Lindl, J.D. and Kaw, P. 1971. Physics of Fluids 14, 371.

12. Hora, H., 2000. Laser Plasma Physics: Forces and the Nonlinearity Principle SPIE-Books, Bellingham, WA.

13. Giuletti, A., Cope, S., Afshar-rad, T., Desselberger, M., Willi, O., Danson, C. And Giulietti, D. 1991. Laser Interaction and Related Plasma Phenomena, H. Hora and G.H. Miley Eds. Plenum, New York, 9: 261.

14. Azechi, H. et al. 1991. Laser and Particle Beams 9, 167.

15. Labaune, C. et al. 1992. Phys. Fluids B 4, 2224,

16. Jackel, S., Barry, B. And Lubin, M. 1976 Phys. Rev. Letters 37, 95.

17. Maddever, R.A.M., 1988. Ph.D. Thesis, Australian National University Canberra.

18. Hora, H. 1975. Laser Fusion and Nuclear Energy (Plenum, New York).

19. Gwynn, P., 1999. Physics World 12 (No. 10).

20. Dawson, J. 2001. Physics Today 54 (No.1) 21.

21. Han-Sheng Peng. 2001. Private Communication, Mianyang.

22. Chiao, R.Y., Garmire, E. And Townes, C.H. 1964. Phys. Rev. Letters 13, 479.

23. Korobkin, V.V. and Alcock, A.J. 1968. Phys. Rev. Letters 21, 1433.

24. Palmer, A.J., 1971. Physics of Fluids, 14, 2714.

25. Shearer, J.W. and Eddleman, J.L. 1973. Physics of Fluids 16, 1753.

26. Kaw, P., Schmidt, G. And Wilcox, T. 1973. Physics of Fluids 16, 1522.

27. Linlor, J. 1963 Appl. Phys. Lett. 3, 210. 
28. Honig, R. E., 1963. Appl. Phys. Lett. 3, 8.

29. Hora, H., 1985. Physics of Fluids 28, 3706.

30. Rowlands, T., 1990. Plasma Physics and Controlled Fusion, 32, 297.

31. Haeuser, T., Scheid, W. And Hora, H. 1992. Physical Review A45, 1278.

32. Hartemann, F.V. et al. 1998. Phys. Rev. E68, 5001.

33. Wang, J.X. et al. 1998. Phys. Rev. E58, 6575.

34. Du, C. And Xu, Z. 1999. Aust. J. Physics 52, 49.

35. Schlüter, A., 1950. Zeitschr. f. Naturforschung 5A, 72.

36. Sklizkov, G.V. 1964. Private Communication. 37.

37. Hora, H. 1967. U.S. Patent No. 3,362,285.

38. Lalousis, P. et al. 1993. Laser and Particle Beams $1,283$.

39. Hora, H., Lalousis, P. And Eliezer, S. 1984. Phys. Rev. Letters 83, 1650.

40. Hora, H. And Ghatak, A.J. 1985. Phys. Rev. A 31, 3473.

41. Goldsworthy, M.P., Hora, H. And Stening, R.J. 1990. Laser and Particle Beams 8, 33.

42. Aleksandrova, W. B. And Sklizkov, G.V. et al. 1985. Laser and Particle Beams 3, 197.

43. Bibeau, C. And Beach, R.J. et al., 2000. SPIE. Proceedings No. 3886, p.57.

44. Eliezer, S. And Hora, H. 1989. Physics Reports, $172,339$.
45. Haseroth, H. And Hora, H. 1996. Laser and Particle Beams 14, 393.

46. Hora, H. 1975a. J. Opt. Soc. Am. 65, 882.

47. Osman, F., Castillo, R. And Hora, H. 1999. J. Plasma Physics, 61263.

48. Hora, H. And Ray, P.S., 1978. Zeitschr. f. Naturforschung A 33, 890.

49. Maddever, R.A.M. and Luther-Davies, B. et al. 1990. Phys. Rev. A41, 215.

50. Hogan, W.J. 2001. Paper 1F/3, Proceedings of the 18th IAEA Fusion Energy Conference, Sorrento, Italy, Oct. 2000 (IAEA CD).

51. Hora, H., Azechi, H., Kitagawa, Y., Mima, K., Murkami, M., Nishihara, K., Takabe, H., Yamanaka, M. And Yamanaka, T. 1998. J. Plasma Physics 60, 743.

52. Lackner, K. And Colgate, S., et al. 1994. Laser Interaction and Related Plasma Phenomena, AIP Conf. Proceedings No. 318, Miley, G.H. Ed. Am. Inst. Phys. New York p. 356.

53. Campbell, E.M., Holmes, N.S., Libby, S.B., Remington, B.A. and Teller, E. 1997. Laser and Particle Beams 15, 607.

54. Tabak, M. et al. 1993. Physics of Plasmas 1, 2010.

55. Boreham, B.W. et al. 1997. Laser and Particle Beams 15, 277. 